\title{
ESTADO DEL ARTE DE RESIDUOS EN MIEL Y CERA EN CHI- LE.
}

\section{CURRENT STATE OF RESIDUES FROM HONEY AND BEESWAX IN CHILE.}

\author{
Nimia Manquián \\ Laboratorio de Fitoquímica, Facultad de Ciencias Agrarias, Universidad Austral de Chile \\ Palabras clave: Miel, cera, residuos, Chile
}

La apicultura en Chile se inicia en 1844 con la importación de abejas desde Italia, iniciativa de grandes propietarios, que no se involucraron en el proceso productivo, delegando la producción a inquilinosotrabajadoressincapacitaciónapícola, formados en la práctica, transformándose así en una actividad complementaria al sustento familiar y en muchos casos heredable.

Es así, como la producción de la miel hasta fines del siglo XX, estuvo en manos de pequeños y medianos apicultores, carentes de capacitación técnica en: producción, comercialización y formas de asociación.

Recién a partir de 1999 se inicia la formación de la Red Nacional Apícola, asociación gremial de pequeños productores, cuyo propósito fundamental fue lograr la sustentabilidad y profesionalización del sector, generando alianzas con entidades académicas, estatales, grandes productores y exportadores.

Actualmente esta organización reúneaproximadamente 2.600 productores - propietarios de 150.000 colmenas, responsables del $35 \%$ de la producción nacional de miel, llegando a ser una de las organizaciones más importantes de la pequeña agricultura chilena.

Entre sus logros se puede destacar:

-La Formación de la Mesa Apícola Nacional (2002), sitio de reunión del sector público y privado, coordinada por la Oficina de Estudios y Políticas Agrarias (ODEPA), dependiente del Ministerio de Agricultura. Un producto de la Mesa Apícola Nacional es el Centro Nacional de Desarrollo Apícola Chile (2004), corporación sin fines de lucro, cuya función es orientar, coordinar y promover el desarrollo de la cadena apícola.
-La asociación con entidades académicas: Pontificia Universidad Católica de Chile y Universidad Austral de Chile, para la ejecución de proyectos relacionados con la calidad, principalmente de la miel y la cera. La asociación con la Universidad Austral de Chile (2003-2007), da origen a un proyecto de diagnóstico de la situación de calidad de las unidades productivas y grado de inocuidad de sus productos (Proyecto Fondo SAG \# 64).

-La difusión de las Buenas Prácticas Agrícolas (BPA) y de Manufactura (BPM) en la Apicultura, auspiciadas por el Ministerio de Agricultura a través de INDAP (Instituto de Desarrollo Agropecuario), a lo largo del país (desde comienzos de 2005) y el inicio de su implementación por los apicultores. La Universidad Austral de Chile y otras instituciones de diverso índole, han participado como ente capacitador en este programa para apicultores del sur de Chile.

Además, a partir del año 2003, se incluye la miel en el Programa de Control de Residuos de Productos Pecuarios de Exportación (de responsabilidad del Servicio Agrícola y Ganadero), con el residuo de fluvalinato, aumentando cada año el control de nuevos residuos, seleccionados sobre la base de la Decisión Comunitaria 97/747, considerando los productos que se utilizan en el manejo apícola nacional y las exigencias de los mercados de destino.

La Universidad Austral de Chile con financiamiento del Fondo de Mejoramiento para el Patrimonio Sanitario, adjudicado por concurso público, ha desarrollado dos proyectos relacionados con la calidad e inocuidad de la miel y la cera desde 1999 a la fecha, encontrándose 
en la actualidad finalizando la última etapa del segundo y en la primera etapa de un tercer proyecto (Fondo SAG \# 23).

El primer proyecto ejecutado por las Universidades Austral de Chile y Católica de Teтисо se denominó: "Acciones sanitarias de prospección, control y vigilancia como bases para un programa de estrategias de manejo integrado de enfermedades en abejas para incrementar la producción de miel en la región de la Araucanía y de Los Lagos" (1999 - 2002), Proyecto Fondo SAG \# 71, teniendo como beneficiarios directos la Cooperativa Campesina Apícola Valdivia, APICOOP Ltda., de la X Región, con 185 productores, y la Sociedad Agro-Cunco Ltda., con 120 productores de la IX, entre las conclusiones de este proyecto se destaca:

"La preocupación causada por la presencia de altos niveles de infestación con varroa, se puede relacionar con los resultados obtenidos en los análisis de algunos residuos. Así, se detectó presencia de residuos de fluvalinato (piretroide), utilizado para el control del ácaro varroa, en cerca del $50 \%$ de las muestras de miel y en cerca del $88 \%$ de las muestras de cera, en niveles, que en promedio, superaron las normas establecidas para este residuo por países importadores de las mieles chilenas, que varía entre 10 y $50 \mu \mathrm{g} / \mathrm{kg}$. En cuanto a los residuos de coumaphos, también utilizado para el control de varroa, fue la IX Región donde se encontró un $42 \%$ de las muestras de miel contaminadas, no así en mieles de la X Región. Por otro lado, en cera la presencia de este plaguicida fue casi nula.

Para ocho tipos de sulfamidas y oxitetraciclina, se analizó una pequeña cantidad de muestras, principalmente con el fin de validar las metodologías. En este contexto, un tercio de las mieles presentó algún tipo de sulfa, en tanto, en ninguna muestra se detectó oxitetraciclina".

Estas conclusiones permitieron formular el segundo proyecto denominado: "Contribución a la sustentabilidad de la apicultura chilena, entre las regiones IV y X a partir del monitoreo de residuos en miel y cera, para incrementar su inocuidad y competitividad de acuerdo a las exigencias de mercado de destino"(2003-2007), teniendo como beneficiarios a los apicultores asociados a la Red Nacional Apícola, entre las regiones IV a X; en este proyecto se aumentó el número de residuos determinados y se inicia el trabajo de terreno con muestreadores capacitados en la Universidad Austral de Chile, para lograr una toma de muestra homogénea en todo el país en apiarios fijos y georreferenciados. El modelo aplicado ha permitido determinar los residuos de productos permitidos o no, para la apicultura a lo largo del país apícola y focalizar las capacitaciones hacia la erradicación del uso de las sustancias prohibidas.

La situación que se mantiene a lo largo del país y que se requiere abordar en el más corto plazo, está relacionada con la industria del proceso de la cera. Los establecimientos que realizan el proceso generalmente no están involucrados en el negocio de la miel, sólo llevan un registro elemental de procedencia, el que está relacionado con fuentes de contaminación microbiana, pero no con los niveles de residuos, tanto del producto que reciben, como del que devuelven en la transacción al apicultor.

El proyecto recientemente iniciado denominado "Contribución a la sustentabilidad de la apicultura en cuatro áreas geográficas de Chile, mediante la determinación del estatus sanitario y su asociación a la calidad de miel y cera" (2007-2010), incluye apicultores que no fueron beneficiados por el proyecto anterior y que representan áreas geográficas aisladas, con un gran potencial de desarrollo de la actividad para obtener productos inocuos y con particulares atributos de calidad para mercados exigentes. (Proyecto Fondo SAG \# 23).

\section{BIBLIOGRAFÍA}

NEIRA, M.; DUSSAUBAT C.; MANQUIAN N. 2002. Informe Final Proyecto Fondo SAG \# 71.

NEIRA, M.; DUSSAUBAT C. (2004, 2005, 2006). Informes Técnicos Proyecto Fondo SAG \# 64.

OYARZUN, M.T.; FIGUEROA, A.; TARTANAC, F. 2005. Oportunidades de Mejoramiento en la Calidad e Inocuidad de la Cadena Productiva de la Miel en Chile. Servicio de Gestión, Comercialización y Finanzas Agrícolas (AGSF). Dirección de Sistemas de Apoyo a la Agricultura. Documento de Trabajo 10. FAO. páginas

SERVICIO AGRICOLA GANADERO 2007. Programa de Control de Residuos de Productos Pecuarios. www.sag.gob.cl 